\title{
Robust Quantum-Network Memory Using Decoherence-Protected Subspaces of Nuclear Spins
}

\author{
Andreas Reiserer, Norbert Kalb, Machiel S. Blok, Koen J. M. van Bemmelen, \\ Tim H. Taminiau, and Ronald Hanson \\ QuTech, Delft University of Technology, P.O. Box 5046, 2600 GA Delft, The Netherlands \\ and Kavli Institute of Nanoscience Delft, Delft University of Technology, \\ P.O. Box 5046, 2600 GA Delft, The Netherlands \\ Daniel J. Twitchen and Matthew Markham \\ Element Six Innovation, Fermi Avenue, Harwell Oxford, Didcot, Oxfordshire OX11 OQR, United Kingdom
}

(Received 9 March 2016; published 22 June 2016)

\begin{abstract}
The realization of a network of quantum registers is an outstanding challenge in quantum science and technology. We experimentally investigate a network node that consists of a single nitrogen-vacancy center electronic spin hyperfine coupled to nearby nuclear spins. We demonstrate individual control and readout of five nuclear spin qubits within one node. We then characterize the storage of quantum superpositions in individual nuclear spins under repeated application of a probabilistic optical internode entangling protocol. We find that the storage fidelity is limited by dephasing during the electronic spin reset after failed attempts. By encoding quantum states into a decoherence-protected subspace of two nuclear spins, we show that quantum coherence can be maintained for over 1000 repetitions of the remote entangling protocol. These results and insights pave the way towards remote entanglement purification and the realization of a quantum repeater using nitrogen-vacancy center quantum-network nodes.
\end{abstract}

DOI: 10.1103/PhysRevX.6.021040

Subject Areas: Quantum Physics, Quantum Information

\section{INTRODUCTION}

Linking multiqubit nodes into a large-scale quantum network [1-4] will open up exciting opportunities ranging from fundamental tests [5] and enhanced timekeeping [6] to applications in quantum computing and cryptography [1,7-9]. Pioneering experiments with atomic ensembles [3], single atoms trapped in vacuum $[2,4,10,11]$, and spins in solids [12-14] have demonstrated entanglement between two optically connected nodes. Directly extending these schemes to quantum networks involving many nodes and spanning large distances is hindered by unavoidable imperfections, including photon loss and local control errors, which cause the success probability and entanglement fidelity to decay rapidly both with the number of nodes and with distance.

These challenges can be overcome via entanglement purification [15] in a repeater-type [16] setting that exploits quantum memories within each node $[7,17,18]$. Crucially, one needs to control and readout individual qubits within the node as well as create entanglement with remote qubits without inducing decoherence on the other qubits in the

*a.a.reiserer@tudelft.nl

Published by the American Physical Society under the terms of the Creative Commons Attribution 3.0 License. Further distribution of this work must maintain attribution to the author(s) and the published article's title, journal citation, and DOI. node. In principle, each of these tasks can be accomplished probabilistically using detectors and quantum memories for single optical photons [19], but the resulting inefficiency poses a severe challenge for practical quantum-network realizations [2,3]. Instead, many efforts are geared towards a layered architecture, as depicted in Fig. 1(a). Here, remote quantum nodes are probabilistically coupled via optical photons, while each node has several deterministically addressable memory qubits that do not interact with and are therefore not disturbed by the optical channel.

A promising candidate for implementing such a quantum-network architecture is the nitrogen-vacancy (NV) center in diamond. The NV electronic spin provides an optical interface that can be used to establish entanglement between distant nodes [13,21,22], while nearby nuclear spins can serve as multiqubit registers [23-26] with second-long coherence times demonstrated in isolation [27]. Here, we address the open challenge of robust simultaneous execution of these two processes-remote entanglement generation and local qubit storage - which is a key prerequisite for entanglement purification and quantum repeaters and therefore a critical task in quantum networks. We implement individual control over five nuclear spin qubits, in which we store quantum states while repeatedly using the electronic spin in a sequence that has previously been used to generate internode entanglement $[13,21,22]$. We study in detail how the fidelity of storage depends on the coupling between electronic and 
(a)
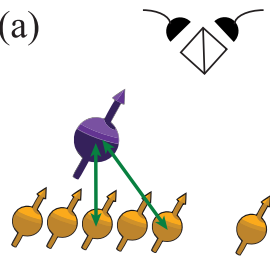

(c)

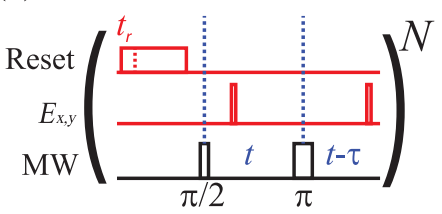

(b)

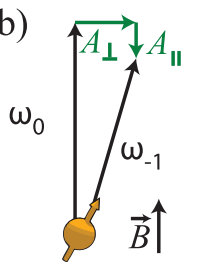

(d)

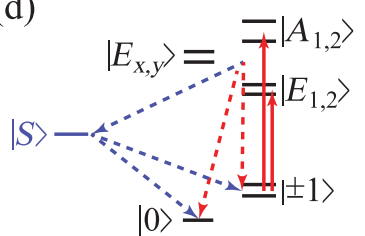

FIG. 1. (a) Layered quantum-network architecture. Individual electronic spins (purple spin symbols) are entangled probabilistically over large distances using photons (red curly lines). Each electronic spin is hyperfine coupled to a quantum register of surrounding nuclear spins (yellow) that can be deterministically controlled (green arrows). (b) Electron-nuclear coupling.

The nuclear spins precess in an external magnetic field $\vec{B}$. The precession axis and frequency, $\omega_{0}$ or $\omega_{-1}$ (black vectors), depend on the state of the electronic spin, $|0\rangle$ or $|-1\rangle$, via the hyperfine interaction with parallel component $A_{\|}$and perpendicular component $A_{\perp}$ (green vectors). (c) Experimental sequence to generate entanglement between remote NV electronic spins [20]. By optical pumping on the "reset" transition, the spin is initialized in $|0\rangle$ at time $t_{r}$. Subsequently, a spin superposition state is created via a microwave $\pi / 2$ pulse. Spin-photon entanglement is then generated via two optical excitations, separated by a microwave $\pi$ pulse that inverts the spin state $|0\rangle \leftrightarrow|-1\rangle$. (d) NV electronic orbital and spin level scheme at cryogenic temperature. The ground states $|0\rangle(| \pm 1\rangle)$ are optically coupled to the excited states $\left|E_{x, y}\right\rangle \quad\left(\left|E_{1,2}\right\rangle\right.$ and $\left|A_{1,2}\right\rangle$, red arrows), respectively. These states decay either directly (red dashed arrows) or via the metastable spin singlet states $|S\rangle$ (blue dashed arrows) to one of the ground states.

nuclear spins and on the average time the electronic spin is in an unknown quantum state. We then use decoherenceprotected subspaces (DPSs) to enhance the robustness of quantum state storage, which enables us to increase the exponential decay constant of the qubit fidelity above 1000 repetitions of the internode entangling sequence.

\section{EXPERIMENTAL SETTING}

The experiments are performed on a diamond device with a natural abundance of ${ }^{13} \mathrm{C}$ nuclear spins $(I=1 / 2$, $\left.m_{I}=1 / 2 \equiv|\uparrow\rangle, m_{I}=-1 / 2 \equiv|\downarrow\rangle\right)$. We cool the device to a temperature of $4 \mathrm{~K}$ in a helium bath cryostat and apply a magnetic field of $40 \mathrm{mT}$ along the NV symmetry axis. Before each experimental run we prepare the NV center in the negative charge state and ensure resonance with the lasers [24]. By using spin-selective optical transitions, the electronic $\operatorname{spin}\left(S=1, m_{s}=0 \equiv|0\rangle, m_{s}= \pm 1 \equiv| \pm 1\rangle\right)$ is initialized with a fidelity above 0.99 and readout in single

shot with an average fidelity of about 0.94 . We employ tailored pulse sequences on the electronic spin $[28,29]$ that yield high-fidelity individual control of five ${ }^{13} \mathrm{C}$ spin qubits surrounding the NV center studied here (the same as in Ref. [29]). In Table I, we list for each nuclear spin qubit the measured hyperfine coupling parameters, the dephasing time $T_{2}^{*}$, and the combined fidelity of spin initialization and readout $F_{i, r}$ (see Supplemental Material [30]).

We now focus on nuclear spin coherence during application of the Barrett-Kok internode entangling sequence [20] [see Fig. 1(c)] that was used in recent experiments $[13,21,22]$. This protocol has two steps. First, entanglement between the electronic spin and the emission time of a single photon is created at both nodes. Subsequently, the two photons are measured after interfering on a beam splitter, probabilistically projecting the electronic spins into a maximally entangled state. Because of unavoidable inefficiencies including photon loss, this sequence has to be repeated many times to generate remote entanglement with a high probability.

Each time an entanglement attempt fails, the electronic spin of the NV center is projected into an unknown state and has to be reset. This can lead to decoherence of the surrounding nuclear spin quantum memories via the (always-on) hyperfine interaction. The interaction Hamiltonian is in secular approximation:

$$
H_{\mathrm{hf}} / 2 \pi=A_{\|} S_{z} I_{z}+A_{\perp} S_{z} I_{x} .
$$

Here, $S$ and $I$ denote the electronic and nuclear spin operators, respectively, and $A_{\|}\left(A_{\perp}\right)$ denote the parallel (perpendicular) hyperfine coupling strength. If the electronic spin state is $|0\rangle$, the nuclear spin will precess at the Larmor frequency $\omega_{0}=2 \pi \times \gamma|\vec{B}|$ around the applied magnetic field $\vec{B}$ [see Fig. 1(b)], where $\gamma=11 \mathrm{kHz} / \mathrm{mT}$ is the nuclear gyromagnetic ratio. If the electronic spin state is $|-1\rangle$, however, the nuclear spin will precess around a slightly tilted axis and at a different frequency, $\omega_{-1}=2 \pi \sqrt{\left(\gamma|\vec{B}|+A_{\|}\right)^{2}+A_{\perp}^{2}}$. In a sufficiently strong

TABLE I. Experimentally determined parameters of the five ${ }^{13} \mathrm{C}$ nuclear spin qubits used in this work: hyperfine couplings $A_{\|}$ and $A_{\perp}$, precession frequency difference $\Delta \omega$, and combined initialization and readout fidelity $F_{i, r}$. The hyperfine parameters are measured via dynamical decoupling spectroscopy [31] and have an uncertainty in the last digit.

\begin{tabular}{lcccccc}
\hline \hline${ }^{13} \mathrm{C}$ & & & & & \\
number & $(\Delta \omega / 2 \pi)(\mathrm{kHz})$ & $A_{\|}(\mathrm{kHz})$ & $A_{\perp}(\mathrm{kHz})$ & $T_{2}^{*}(\mathrm{~ms})$ & $F_{i, r}$ \\
\hline 1 & -15.4 & -11.0 & 55 & $6(1)$ & $0.89(2)$ \\
2 & 18.4 & 21.2 & 43 & $13(1)$ & $0.96(2)$ \\
3 & 23.7 & 24.7 & 26 & $19(2)$ & $0.97(2)$ \\
4 & -37.0 & -36.0 & 25 & $10(1)$ & $0.92(2)$ \\
5 & -48.6 & -48.7 & 12 & $4(1)$ & $0.90(2)$ \\
\hline \hline
\end{tabular}


magnetic field, $\gamma|\vec{B}| \gg \sqrt{A_{\perp}^{2}+A_{\|}^{2}}$, the change in precession axis is quadratically suppressed and nuclear spin decoherence is mainly caused by dephasing due to the linear change in the precession frequency:

$$
\Delta \omega=\omega_{0}-\omega_{-1} \simeq 2 \pi \times A_{\|} .
$$

Thus, randomization of the electronic spin state is expected to lead to dephasing of a nuclear spin on a time scale that is inversely proportional to the parallel hyperfine coupling strength $[32,33]$.

This dephasing can be mitigated by a suited dynamical decoupling sequence [33], which is inherent in the BarrettKok entangling sequence [Fig. 1(c)]: for $\tau=0$, the time interval between the microwave (MW) $\pi / 2$ pulse and the MW $\pi$ pulse has the same duration as the time interval between the MW $\pi$ pulse and the start of the electronic spin reset. Thus, the electronic spin will be in $|0\rangle$ and $|-1\rangle$ for an equal amount of time, irrespective of the random optical projection. Thus, under the condition that the spin reset is instantaneous and happens at a precisely known time, the dephasing is exactly nullified. However, electronic spin reset by optical pumping is a stochastic process of finite time duration. As the spin state is not known during this process, it induces irreversible dephasing of the nuclear spins. Therefore, it is desirable to use nuclear spins with weak coupling strength and to make the electronic spin reset as short as possible.

\section{ELECTRONIC SPIN RESET}

We first investigate the spin reset process and optimize the reset time. We initialize the electronic spin in $|0\rangle$ by applying laser fields that are on resonance either with the transitions $|-1\rangle \leftrightarrow\left|E_{1}\right\rangle$ and $|+1\rangle \leftrightarrow\left|E_{2}\right\rangle$ or, for comparison, with the transitions $|-1\rangle \leftrightarrow\left|A_{1}\right\rangle$ and $|+1\rangle \leftrightarrow$ $\left|A_{2}\right\rangle$; see Fig. 1(d). Compared to our previous remote entanglement experiments [13,21,22], the use of higher magnetic fields requires a second laser beam because of the comparably large ground state Zeeman splitting between $|-1\rangle$ and $|+1\rangle$ of about $2 \mathrm{GHz}$. The lasers address different excited states to avoid pumping to a coherent dark state. Both repump laser beams have approximately circular polarization and equal power. The excited states have a lifetime on the order of $10 \mathrm{~ns}$ [34]. From the excited states, the NV can decay either back to $| \pm 1\rangle$ or to the metastable spin singlet states $|S\rangle$. In addition, spin mixing in the excited states also opens a direct decay channel to $|0\rangle$ [35].

To determine the time it takes to reset the spin, we prepare it in $|-1\rangle$ and pump it with the reset lasers for a varying duration. After a delay of $2.5 \mu$ s to ensure that no population is left in the excited or singlet states [35], we measure the population in $|0\rangle$; see Fig. 2(a). The spin pumping process exhibits a double-exponential decay with a relative amplitude ratio for the fast and slow time scales of (a)

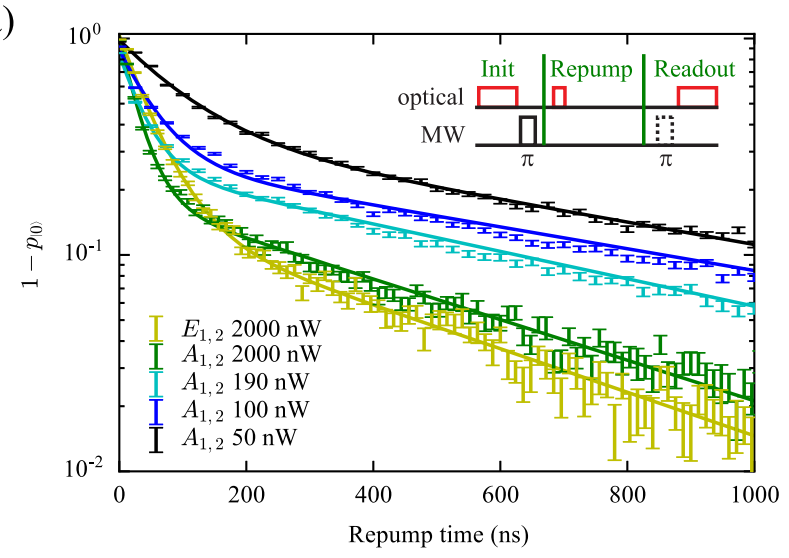

(b)
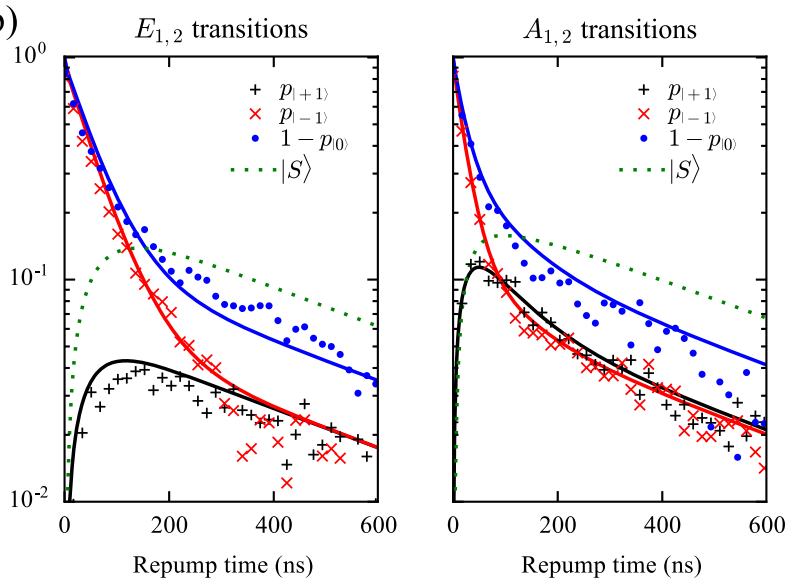

FIG. 2. NV electronic spin initialization. (a) Probability that the electronic spin is pumped to $|0\rangle$ as a function of the repump laser pulse duration when the spin is initially prepared in $|-1\rangle$. The inset shows the used pulse sequence. We observe a doubleexponential decay (solid fit curves), with a time scale and relative amplitude that depends on the used "reset" transition. Reduced laser power leads to slower initialization time scales. (b) Probability that the NV is found in state $|0\rangle,|+1\rangle$, and $|-1\rangle$ for 2000-nW repump power in the $\left|E_{1,2}\right\rangle$ (left) or $\left|A_{1,2}\right\rangle$ (right) configuration. The solid lines are calculated using a rate equation model described in the text. For long repumping time, the calculated population in the metastable singlet states (green dotted curve) dominates the repumping process.

around $3: 1$, which slightly depends on the excited states used. The two time scales of this decay depend on the applied laser power. At high power, the reset time scales saturate (see Supplemental Material [30]) at 29(1) and 463(14) ns when driving transitions to $\left|A_{1,2}\right\rangle$ [green, Fig. 2(a)] and at 48(1) and 432(26) ns when driving transitions to $\left|E_{1,2}\right\rangle$ [yellow, Fig. 2(a)].

We attribute the slower time scale, which is the same for both configurations within measurement uncertainty, to the decay constant of the metastable singlet states. The fitted value is in the same range as previously reported values [36]. The faster time scale has a contribution from both direct spin-flip transitions to $|0\rangle$ and transitions into the 
singlet states which then decay to $|0\rangle$. The difference in fast time scales between the two configurations is explained by different decay rates to the ground states and metastable singlet states from the excited states used [34]. When the laser power is reduced, we observe a gradual increase of both time scales, as shown for the $\left|A_{1,2}\right\rangle$ configuration [cyan, blue, and black curves in Fig. 2(a)].

To obtain additional insight into the spin reset process, we measure the probabilities $p_{i}$ to arrive in the states $i=|0\rangle,|-1\rangle$, and $|+1\rangle$, again $2.5 \mu$ s after applying a repump pulse of varying duration. In Fig. 2(b), we plot $1-p_{|0\rangle}, p_{|-1\rangle}$, and $p_{|+1\rangle}$. We fit the data to rate equation models (solid lines), one for each repump configuration. These models assume the lifetimes of the individual states as measured in Ref. [34], a lifetime of the metastable singlet states of $440 \mathrm{~ns}$ (taken from the above fits), a relative singlet decay ratio $R_{i}$ to the states $i$ of $\left(R_{|0\rangle}: R_{|+1\rangle}: R_{|-1\rangle}\right)=$ $(2: 1: 1)$ [35], and full decay of the singlet and excited states before the ground state population is measured. The model uses equal decay of both excited states $\left|A_{1,2}\right\rangle$ to $| \pm 1\rangle$, while the states $\left|E_{1,2}\right\rangle$ decay either to $|+1\rangle$ or to $|-1\rangle$ [35]. The decay rates of the excited states to $|0\rangle$ and the rate of excitation and stimulated emission caused by the repump laser are free parameters in the model. The quantitative agreement between data and the model strengthens the hypothesis that the slow time scale of the repump process is dominated by the lifetime of the metastable singlet states. The population of the metastable singlet states (before decay to the ground states) predicted by the models is shown as the green dotted curve [Fig. 2(b)].

\section{SINGLE-NUCLEAR-SPIN QUANTUM MEMORY}

We now turn to the dephasing of single-nuclear-spin qubits induced by the stochastic trajectory of the electronic spin state during reset. Using recently developed techniques [29], we first initialize one of the five controlled nuclear spins by a projective measurement (see Supplemental Material [30]). Ideally, this prepares the nuclear spin superposition state $\frac{1}{\sqrt{2}}(|\downarrow\rangle+|\uparrow\rangle)$. We then perform $N$ repetitions of the remote entanglement sequence. We omit the short optical $\pi$ pulses, as they are expected to induce negligible nuclear spin dephasing since they preserve the electronic spin eigenstate and can be timed such that the detrimental effect of occasional spin flips ( $p_{\text {flip }}<1 \%$ ) [24] is mitigated by the dynamical decoupling sequence. In addition, the fast optical pulses only lead once per sequence to a population of the excited state, whose spin projection is zero and whose 12-ns lifetime is short compared to the reset procedure. We track the dephasing of the nuclear spins by measuring the length of their Bloch vector projection onto the $X Y$ plane. We do not include the $Z$ projection, as it remains small. In addition, we discard the small fraction of experimental runs in which the NV electronic spin is ionized (Supplemental Material [30]).

We first investigate and optimize the timings $t$ and $\tau$ of the dynamical decoupling sequence shown in Fig. 1(c). We find that the dephasing rate shows a clear minimum when $t$ equals the Larmor period of the nuclear spins (see Supplemental Material [30]), in which case the effect of entanglement between electronic and nuclear spins caused by the tilted rotation axis, as shown in Fig. 1(b), is minimized. We therefore set $t=\left(2 \pi / \omega_{0}\right) \simeq 2.3 \mu \mathrm{s}$. We then repeat the entanglement sequence $N=200$ times and vary the time $\tau$. Assuming an exponential distribution of the repumping time $t_{r}$, one expects to obtain the best possible decoupling when $\tau$ is equal to $\left\langle t_{r}\right\rangle$ [33]. As can be seen in Fig. 3(a), we observe a broad Gaussian distribution centered around an optimal value of $\tau \approx 0.44 \mu$ s for all four measured nuclear spins, in good agreement with the slow time scale of the initialization process shown in Fig. 2(a). As mentioned, we attribute this time scale to the decay of the metastable singlet states. At first sight, it is surprising that a singlet state which has zero spin projection and thus no hyperfine coupling would induce dephasing. A possible explanation is that the orbital angular momentum of an $E$-symmetric singlet state induces a magnetic moment that is comparable to that of the electronic spin ground states.

Additional dephasing can result from experimental imperfections. To prevent errors caused by imperfect spin initialization, e.g., when the laser power drifts over time, we apply the repump laser longer than required for the initialization curves to saturate below 0.01 , which is $2 \mu \mathrm{s}(1.5 \mu \mathrm{s})$ for the $\left|E_{1,2}\right\rangle\left(\left|A_{1,2}\right\rangle\right)$ repump configuration at $2000 \mathrm{nW}$, respectively. To prevent errors caused by imperfect MW pulses, we employ a Hermite pulse envelope with a broad spectrum in order to drive the $|0\rangle \leftrightarrow|-1\rangle$ transition irrespective of the spin state of the nitrogen nucleus of the NV center. We employ this pulse in a BB1 composite pulse sequence [37], consisting of five pulses of less than $0.2 \mu$ s duration each, to further suppress pulse errors. In this way, we are able to exclude experimental imperfections as a relevant source of the observed dephasing (see Supplemental Material for details [30]).

With the optimized timings and pulses, we then vary the number of repetitions $N$ of the remote entanglement sequence. For all five nuclear spins, we observe an exponential decay of the single-qubit coherence with $N$; see Fig. 3(b). Even for the nuclear spin with the largest parallel hyperfine coupling - for which the dephasing is fastest-more than $N=100$ repetitions of the entanglement sequence can be run before the Bloch vector length drops to $1 / e$. For the nuclear spin with the smallest coupling strength, this number increases to $N \approx 500$. Further improvements could be achieved by using nuclear spins with even lower parallel hyperfine coupling, although 

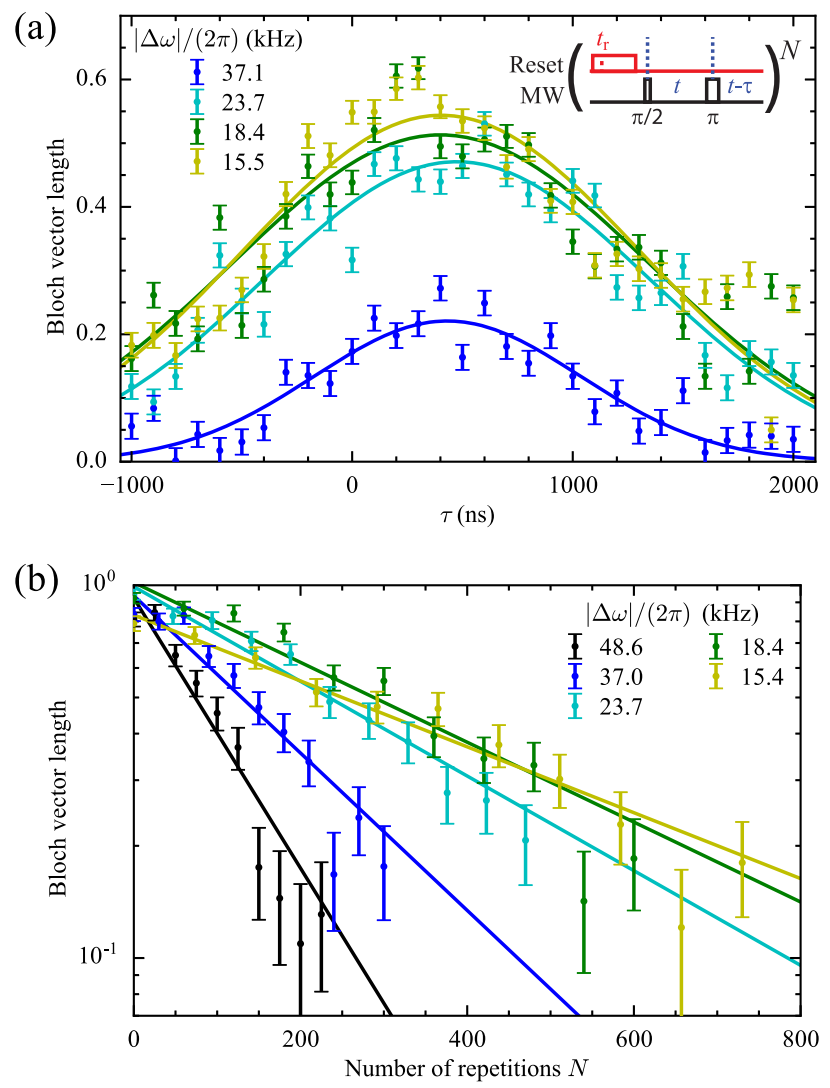

FIG. 3. Dephasing of ${ }^{13} \mathrm{C}$ nuclear spins. (a) Optimization of the dynamical decoupling sequence timing. Different nuclear spins (colors) are initialized in a balanced superposition state and the entanglement sequence is performed $N=200$ times. The duration of the second wait interval is swept and the length of the Bloch vector $X Y$ projection is measured. All measured nuclear spins exhibit the same broad optimum around $0.4 \mu \mathrm{s}$, as can be seen from the Gaussian fit curves. (b) Dephasing of nuclear spins when the number of random reset processes is increased. The data show measurement results of all five individually controlled nuclear spins, where increasing coupling strength leads to steeper decay curves. The solid lines are exponential fits.

this would generally come at the price of an increased duration of local control operations.

\section{DECOHERENCE-PROTECTED SUBSPACE QUANTUM MEMORY}

Motivated by the observation that the memory dephasing is mainly determined by the parallel hyperfine coupling strength, we investigate a different approach to increasing the maximum number of repetitions before a qubit is dephased. Instead of encoding the qubit in a single nuclear spin, we can encode in a decoherence-protected subspace [38] of two or more nuclear spins. In this way, the net parallel hyperfine coupling can be strongly reduced while the speed of the individual quantum gates remains the same.
A natural choice for a DPS with reduced dephasing is given by the basis states $\left|\downarrow_{i} \uparrow_{j}\right\rangle$ and $\left|\uparrow_{i} \downarrow_{j}\right\rangle$ of nuclear spins $i$ and $j$ with comparable parallel hyperfine coupling strength. An encoded qubit will then, to first order, dephase at a rate that is determined by the coupling strength difference $\Delta \omega \simeq 2 \pi\left(A_{\|, i}-A_{\|, j}\right)$, which can be much smaller than the individual coupling strengths. On the other hand, encoding a qubit in the states $\left|\uparrow_{i} \uparrow_{j}\right\rangle$ and $\left|\downarrow_{i} \downarrow_{j}\right\rangle$ will lead to increased dephasing rates.

To experimentally verify these expectations, we create the states $\left(\left|\downarrow_{i} \uparrow_{j}\right\rangle+\left|\uparrow_{i} \downarrow_{j}\right\rangle\right) / \sqrt{2}$ and $\left(\left|\uparrow_{i} \uparrow_{j}\right\rangle+\left|\downarrow_{i} \downarrow_{j}\right\rangle\right) / \sqrt{2}$ [29] (see Supplemental Material [30]) and measure the qubit state projection onto the $X Y$ plane of the Bloch sphere under the remote entangling protocol. Figure 4(a)
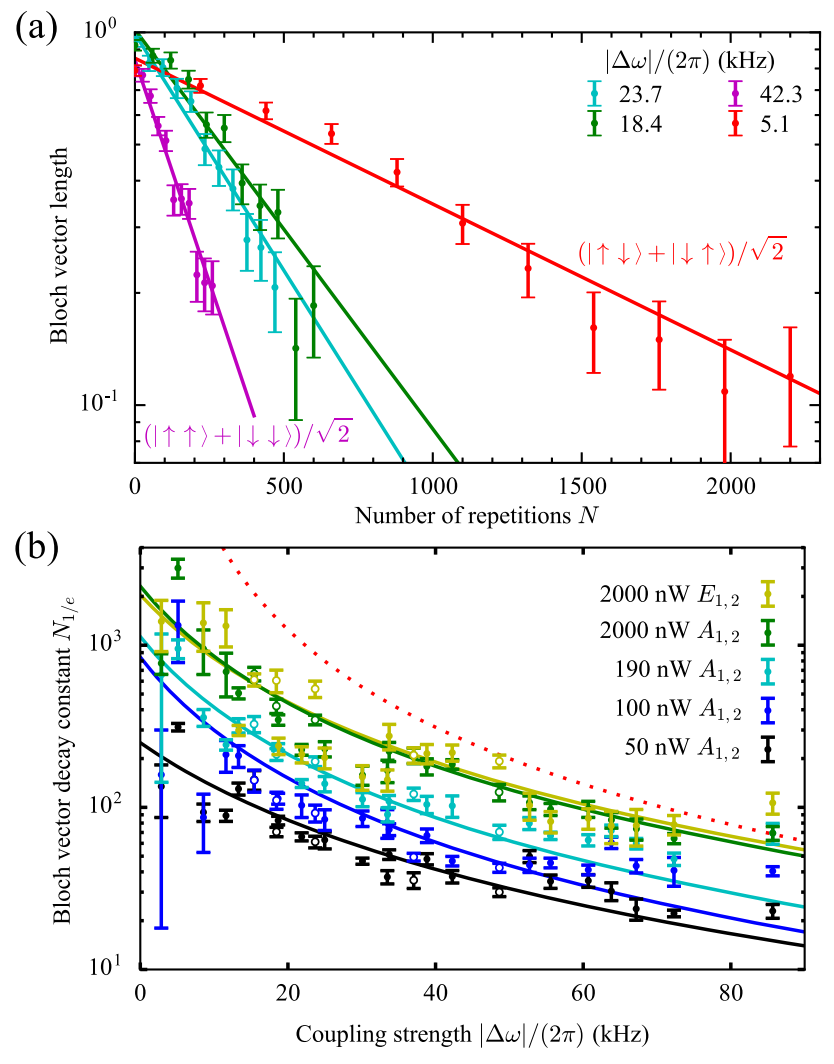

FIG. 4. Encoding of a quantum bit in decoherence-protected subspaces. (a) Encoding in nuclear spin 2 or 3 (cyan and green) shows similar decay with increasing $N$. Encoding in a decoherence-protected (decoherence-enhanced) subspace leads to strongly decreased (enhanced) dephasing shown in red (magenta). The initial fidelity in the two-spin case is slightly reduced because encoding and readout require more control operations on the nuclear spins. (b) Number of sequence repetitions that are possible before the nuclear qubit Bloch vector length drops to $1 / e$ of its initial value, for qubits encoded in single nuclear spins (empty circles) and in two-spin states (filled circles) of different effective coupling strengths $\Delta \omega$. The four depicted data sets are taken for increasing repump duration, caused by a reduced repump laser power. The solid curves are fits according to the model we present in the text. 
shows the results obtained for nuclear spins 2 and 3 . When initializing the qubit in a decoherence-protected (decoherence-enhancing) two-spin state, we observe a strong improvement (reduction) of the maximum number of repetitions. In the DPS case, we can perform more than 1000 repetitions before the Bloch vector length drops to $1 / e$. This decay constant can be fully explained by the intrinsic dephasing time $T_{2}^{*}$ of the nuclear spins (see Supplemental Material [30]). This shows that the dephasing induced by the entanglement protocol has become negligible in this DPS quantum memory.

The coherence of a DPS with small effective coupling strength might also be limited by the population decay $\left(T_{1}\right)$ of the individual spins induced by the entanglement protocol. For the current sample, we find an exponential decay of population with $N$ with decay constants in the range of 1000 to 10000 repetitions, depending on the individual nuclear spins used (see Supplemental Material [30]). We note that this effect is not limiting the coherence of the two-qubit DPS we investigate here, but we expect it to become relevant for the smallest investigated coupling strengths once the intrinsic dephasing (with $T_{2}^{*}$ times of a few milliseconds in the current sample; see Table I) is canceled by an echo.

\section{SCALING OF THE DEPHASING RATE WITH HYPERFINE COUPLING STRENGTH AND REPUMP DURATION}

Finally, we perform an extensive quantitative investigation of the scaling of dephasing with the coupling strength and with the time it takes to reset the electronic spin. Figure 4(b) shows the number of entanglement sequence repetitions $N_{1 / e}$ for which the state fidelity of a balanced superposition state decays to $1 / e$ of the initial value [i.e., the fitted decay constant in Fig. 4(a)]. We investigate all five individual nuclear spins (open circles) and all 20 possible two-spin subspaces (filled circles), whose coupling strength is the sum or difference of the individual ones. The depicted five data sets correspond to the different values of the repumping time constants shown in Fig. 2. To ensure that we investigate only the scaling of the dephasing with coupling strength and repump duration, we correct for the effects of $T_{2}^{*}$ decay, which becomes dominant for the leftmost three data points (see Supplemental Material [30]) and could be compensated by a suited echo sequence on the nuclear spins [27].

We compare the data to the model of Blok et al. [33], which assumes an exponentially distributed repump time scale and that the NV stays in the ground state $|-1\rangle$ until it is reset. When the value of $\tau$ used in the dynamical decoupling sequence is equal to the average repump time $\left\langle t_{r}\right\rangle$, a nuclear spin has acquired a phase shift of $\Delta \omega\left(t_{r}-\tau\right)$ until the electronic spin is reset to $|0\rangle$. In the limit of large $N$, the binomial probability distribution of required electronic spin resets can be approximated by a Gaussian distribution. For a balanced superposition input state, as we investigate here, this leads to a predicted qubit fidelity of

$$
F=\frac{1}{2}+\frac{1}{2^{N+1}}\left(1+e^{-\Delta \omega^{2} \tau^{2} / 2}\right)^{N} .
$$

Thus, the model correctly predicts the observed exponential dephasing of the qubit with increasing number of repetitions $N$. As expected, the decay constant depends on the hyperfine coupling strength of the nuclear spin with a faster decay for increased coupling strength. However, the prediction of the model when inserting the measured optimal value of $\tau=0.44 \mu$ s [red dotted line, Fig. 4(b)] does not exhibit quantitative agreement with the measured data. A possible explanation is that the model neglects the double-exponential reset time distribution and the time spent in one of the excited states, the metastable singlet states or in $|+1\rangle$, in each of which the hyperfine coupling and, therefore, the nuclear spin precession frequency can be different. These assumptions of the model are certainly not justified in the present experiment.

To account for this, we leave $\tau$ in Eq. (3) as a free parameter and introduce an offset parameter $C$ to the coupling strength, $\Delta \omega \rightarrow(\Delta \omega+C)$. Setting $C$ to $\approx 2 \pi \times$ $15 \mathrm{kHz}$ leads to reasonable agreement of the model [solid curves in Fig. 4(b)] with the data. The observed fit values $\left[\tau=0.43(3) \mu \mathrm{s}\right.$ for the $\left|E_{1,2}\right\rangle$ and $\tau=0.46(1) \mu \mathrm{s}$ for the $\left|A_{1,2}\right\rangle$ repumping configuration] agree within error with both the measured slow repumping time scale, see Fig. 2(a), and the optimal value of $\tau$ in Fig. 3(a).

\section{CONCLUSION AND OUTLOOK}

We study a prototype quantum-network node consisting of nuclear spin qubits hyperfine coupled to an optically active electronic spin in a diamond with natural isotope abundance. Repeated application of a remote entangling protocol is observed to cause dephasing of the nuclear spin qubits. We find that this dephasing can be mitigated by using nuclear spins with small parallel hyperfine coupling strengths, and, even more effectively, by encoding in decoherence-protected subspaces of multiple spins. For the smallest coupling strengths investigated here, the storage of quantum states is robust to more than 1000 remote entangling attempts. We expect that our experimental findings can be generalized to other physical systems [39] and other quantum protocols in which a repeated reset of an ancilla qubit with always-on coupling to a memory is required.

In the future, the implementation of high-quality optical cavities should allow for a further reduction of the time it takes to reset the electronic spin, as the Purcell effect [40] induced by such a resonator increases the probability of direct spin flips without populating the singlet states. In addition, the development of techniques to measure the electronic spin state nondestructively or within a 
decoupling sequence might fully eliminate the need for probabilistic repumping. Finally, the realization of quantum-networking protocols that are based on photon absorption [41-43] rather than photon emission may reduce the number of required electronic spin resets until a successful entanglement event is heralded.

Even in the absence of such future improvements, the measured number of remote entangling attempts that the memory can withstand $(>1000)$ is comparable to the number of attempts required to detect one entangling photon (about $10^{-3}$ in Ref. [22]). We therefore anticipate that the current results will enable first demonstrations of the purification of remote entanglement $[15,44]$ and proofof-principle operation of a quantum repeater [16] based on NV centers in diamond [17].

\section{ACKNOWLEDGMENTS}

We thank Adam Gali for discussions on the NV singlet states. We acknowledge support from the Dutch Organization for Fundamental Research on Matter (FOM), the Netherlands Organization for Scientic Research (NWO) through a VENI grant (T.H. T.), and the European Research Council through a Starting Grant (R. H.).

A. R. and N. K. contributed equally to this work.

[1] H. J. Kimble, The Quantum Internet, Nature (London) 453, 1023 (2008).

[2] L.-M. Duan and C. Monroe, Colloquium: Quantum Networks with Trapped Ions, Rev. Mod. Phys. 82, 1209 (2010).

[3] N. Sangouard, C. Simon, H. de Riedmatten, and N. Gisin, Quantum Repeaters Based on Atomic Ensembles and Linear Optics, Rev. Mod. Phys. 83, 33 (2011).

[4] A. Reiserer and G. Rempe, Cavity-Based Quantum Networks with Single Atoms and Optical Photons, Rev. Mod. Phys. 87, 1379 (2015).

[5] J.-D. Bancal, S. Pironio, A. Acín, Y.-C. Liang, V. Scarani, and N. Gisin, Quantum Non-Locality Based on Finite-Speed Causal Influences Leads to Superluminal Signalling, Nat. Phys. 8, 867 (2012).

[6] P. Kómár, E. M. Kessler, M. Bishof, L. Jiang, A. S. Sørensen, J. Ye, and M. D. Lukin, A Quantum Network of Clocks, Nat. Phys. 10, 582 (2014).

[7] N. H. Nickerson, Y. Li, and S.C. Benjamin, Topological Quantum Computing with a Very Noisy Network and Local Error Rates Approaching One Percent, Nat. Commun. 4, 1756 (2013).

[8] S. Barz, E. Kashefi, A. Broadbent, J. F. Fitzsimons, A. Zeilinger, and P. Walther, Demonstration of Blind Quantum Computing, Science 335, 303 (2012).

[9] A. Ekert and R. Renner, The Ultimate Physical Limits of Privacy, Nature (London) 507, 443 (2014).

[10] J. Hofmann, M. Krug, N. Ortegel, L. Gérard, M. Weber, W. Rosenfeld, and $\mathrm{H}$. Weinfurter, Heralded Entanglement between Widely Separated Atoms, Science 337, 72 (2012).
[11] D. Hucul, I. V. Inlek, G. Vittorini, C. Crocker, S. Debnath, S. M. Clark, and C. Monroe, Modular Entanglement of Atomic Qubits Using Photons and Phonons, Nat. Phys. 11, 37 (2015).

[12] W. B. Gao, A. Imamoglu, H. Bernien, and R. Hanson, Coherent Manipulation, Measurement and Entanglement of Individual Solid-State Spins Using Optical Fields, Nat. Photonics 9, 363 (2015).

[13] B. Hensen, H. Bernien, A. E. Dréau, A. Reiserer, N. Kalb, M. S. Blok, J. Ruitenberg, R. F. L. Vermeulen, R. N. Schouten, C. Abellán, W. Amaya, V. Pruneri, M. W. Mitchell, M. Markham, D. J. Twitchen, D. Elkouss, S. Wehner, T. H. Taminiau, and R. Hanson, Loophole-Free Bell Inequality Violation Using Electron Spins Separated by 1.3 Kilometres, Nature (London) 526, 682 (2015).

[14] A. Delteil, Z. Sun, W.-bo Gao, E. Togan, S. Faelt, and A. Imamoğlu, Generation of Heralded Entanglement between Distant Hole Spins, Nat. Phys. 12, 218 (2016).

[15] C. H. Bennett, G. Brassard, S. Popescu, B. Schumacher, J. A. Smolin, and W. K. Wootters, Purification of Noisy Entanglement and Faithful Teleportation via Noisy Channels, Phys. Rev. Lett. 76, 722 (1996).

[16] H.-J. Briegel, W. Dür, J. I. Cirac, and P. Zoller, Quantum Repeaters: The Role of Imperfect Local Operations in Quantum Communication, Phys. Rev. Lett. 81, 5932 (1998).

[17] L. Childress, J. M. Taylor, A. S. Sørensen, and M. D. Lukin, Fault-Tolerant Quantum Communication Based on SolidState Photon Emitters, Phys. Rev. Lett. 96, 070504 (2006).

[18] A. G. Fowler, D. S. Wang, C. D. Hill, T. D. Ladd, R. Van Meter, and L. C. L. Hollenberg, Surface Code Quantum Communication, Phys. Rev. Lett. 104, 180503 (2010).

[19] L.-M. Duan, M. D. Lukin, J. I. Cirac, and P. Zoller, LongDistance Quantum Communication with Atomic Ensembles and Linear Optics, Nature (London) 414, 413 (2001).

[20] S. D. Barrett and P. Kok, Efficient High-Fidelity Quantum Computation Using Matter Qubits and Linear Optics, Phys. Rev. A 71, 060310 (2005).

[21] H. Bernien, B. Hensen, W. Pfaff, G. Koolstra, M. S. Blok, L. Robledo, T. H. Taminiau, M. Markham, D. J. Twitchen, L. Childress, and R. Hanson, Heralded Entanglement between Solid-State Qubits Separated by Three Metres, Nature (London) 497, 86 (2013).

[22] W. Pfaff, B. J. Hensen, H. Bernien, S. B. van Dam, M. S. Blok, T. H. Taminiau, M. J. Tiggelman, R. N. Schouten, M. Markham, D. J. Twitchen, and R. Hanson, Unconditional Quantum Teleportation between Distant Solid-State Quantum Bits, Science 345, 532 (2014).

[23] M. V. Gurudev Dutt, L. Childress, L. Jiang, E. Togan, J. Maze, F. Jelezko, A. S. Zibrov, P. R. Hemmer, and M. D. Lukin, Quantum Register Based on Individual Electronic and Nuclear Spin Qubits in Diamond, Science 316, 1312 (2007).

[24] L. Robledo, L. Childress, H. Bernien, B. Hensen, P. F. A. Alkemade, and R. Hanson, High-Fidelity Projective ReadOut of a Solid-State Spin Quantum Register, Nature (London) 477, 574 (2011).

[25] G.-Q. Liu, H. C. Po, J. Du, R.-B. Liu, and X.-Yu Pan, NoiseResilient Quantum Evolution Steered by Dynamical Decoupling, Nat. Commun. 4, 2254 (2013). 
[26] G. Waldherr, Y. Wang, S. Zaiser, M. Jamali, T. SchulteHerbrüggen, H. Abe, T. Ohshima, J. Isoya, J. F. Du, P. Neumann, and J. Wrachtrup, Quantum Error Correction in a Solid-State Hybrid Spin Register, Nature (London) 506, 204 (2014).

[27] P. C. Maurer, G. Kucsko, C. Latta, L. Jiang, N. Y. Yao, S. D. Bennett, F. Pastawski, D. Hunger, N. Chisholm, M. Markham, D. J. Twitchen, J. I. Cirac, and M. D. Lukin, Room-Temperature Quantum Bit Memory Exceeding One Second, Science 336, 1283 (2012).

[28] T. H. Taminiau, J. Cramer, T. van der Sar, V. V. Dobrovitski, and R. Hanson, Universal Control and Error Correction in Multi-Qubit Spin Registers in Diamond, Nat. Nanotechnol. 9, 171 (2014).

[29] J. Cramer, N. Kalb, M. Adriaan Rol, B. Hensen, M. S. Blok, M. Markham, D. J. Twitchen, R. Hanson, and T. H. Taminiau, Repeated Quantum Error Correction on a Continuously Encoded Qubit by Real-Time Feedback, Nat. Commun. 7, 11526 (2016).

[30] See Supplemental Material at http://link.aps.org/ supplemental/10.1103/PhysRevX.6.021040 for further experimental details, including the nuclear spin initialization and readout procedure, optimization of the sequence timing, the influence of imperfections, the power dependence of the optical pumping procedure, the probability of NV deionization, and nuclear spin polarization decay.

[31] T. H. Taminiau, J. J. T. Wagenaar, T. van der Sar, F. Jelezko, V. V. Dobrovitski, and R. Hanson, Detection and Control of Individual Nuclear Spins Using a Weakly Coupled Electron Spin, Phys. Rev. Lett. 109, 137602 (2012).

[32] L. Jiang, M. V. G. Dutt, E. Togan, L. Childress, P. Cappellaro, J. M. Taylor, and M. D. Lukin, Coherence of an Optically Illuminated Single Nuclear Spin Qubit, Phys. Rev. Lett. 100, 073001 (2008).

[33] M. S. Blok, N. Kalb, A. Reiserer, T. H. Taminiau, and R. Hanson, Towards Quantum Networks of Single Spins: Analysis of a Quantum Memory with an Optical Interface in Diamond, Faraday Discuss. 184, 173 (2015).
[34] M. L. Goldman, A. Sipahigil, M. W. Doherty, N. Y. Yao, S. D. Bennett, M. Markham, D. J. Twitchen, N. B. Manson, A. Kubanek, and M. D. Lukin, Phonon-Induced Population Dynamics and Intersystem Crossing in Nitrogen-Vacancy Centers, Phys. Rev. Lett. 114, 145502 (2015).

[35] M. W. Doherty, N. B. Manson, P. Delaney, F. Jelezko, J. Wrachtrup, and L. C. L. Hollenberg, The Nitrogen-Vacancy Colour Centre in Diamond, Phys. Rep. 528, 1 (2013).

[36] L. Robledo, H. Bernien, I. van Weperen, and R. Hanson, Control and Coherence of the Optical Transition of Single Nitrogen Vacancy Centers in Diamond, Phys. Rev. Lett. 105, 177403 (2010).

[37] L. M. K. Vandersypen and I. L. Chuang, NMR Techniques for Quantum Control and Computation, Rev. Mod. Phys. 76, 1037 (2005).

[38] D. A. Lidar, I. L. Chuang, and K. B. Whaley, DecoherenceFree Subspaces for Quantum Computation, Phys. Rev. Lett. 81, 2594 (1998).

[39] D. D. Awschalom, L. C. Bassett, A. S. Dzurak, E. L. Hu, and J. R. Petta, Quantum Spintronics: Engineering and Manipulating Atom-like Spins in Semiconductors, Science 339, 1174 (2013).

[40] E. M. Purcell, Spontaneous Emission Probabilities at Radio Frequencies, Phys. Rev. 69, 681 (1946).

[41] N. Kalb, A. Reiserer, S. Ritter, and G. Rempe, Heralded Storage of a Photonic Quantum Bit in a Single Atom, Phys. Rev. Lett. 114, 220501 (2015).

[42] S. Yang, Ya Wang, D. D. Bhaktavatsala Rao, T. H. Tran, S. A. Momenzadeh, R. Nagy, M. Markham, D. J. Twitchen, P. Wang, W. Yang, R. Stoehr, P. Neumann, H. Kosaka, and J. Wrachtrup, High Fidelity Transfer and Storage of Photon States in a Single Nuclear Spin, arXiv:1511.04939.

[43] C. Jones, D. Kim, M. T. Rakher, P. G. Kwiat, and T. D. Ladd, Design and Analysis of Communication Protocols for Quantum Repeater Networks, arXiv:1505.01536.

[44] E. T. Campbell and S. C. Benjamin, Measurement-Based Entanglement under Conditions of Extreme Photon Loss, Phys. Rev. Lett. 101, 130502 (2008). 